\title{
Uses of a familial adenomatous polyposis registry
}

\author{
K U A Dalpatadu', N Anwar', S R E Wijesuriya ${ }^{1}$, S K Kumarage'1, B Amarasinghe ${ }^{2}$, K I Deen ${ }^{1}$
}

(Index words: familial adenomatous polyposis, colorectal cancer, polyposis register)

\begin{abstract}
Objectives To improve the prognosis of patients with familial adenomatous polyposis (FAP) by early diagnosis and prophylactic treatment through a coordinated FAP register.
\end{abstract}

Design The establishment and descriptive analysis of the prospective database of the FAP registry.

Setting University surgical unit, Colombo North Teaching Hospital Ragama, Sri Lanka.

Patients Probands were identified by tracing all diagnosed FAP patients from 1996 to 2010 and their family members at risk.

Interventions The establishment of a polyposis register included the following stages: ascertainment of probands (first contact symptomatic FAP patients), construction of pedigrees, counselling relatives and prophylactic screening of family members at risk, treatment and follow up.

Results Twenty seven enrolled probands (12 male and 15 female, age 11-52 years, median age 34 years) were investigated. Pedigree analyses showed 206 relatives at risk. Twenty four family members at risk were screened of a total of 51 registered individuals. The rate of spontaneous mutations was $41 \%$. Thirty five were diagnosed with FAP. Eight were screen detected (median age -32 years) and 27 symptomatic (median age - 34 years). Concomitant colorectal cancer was detected in $17(63 \%)$ symptomatic individuals and in $1(13 \%)$ screen detected individual. Colectomy was performed in 27 (77\%) patients while $8(23 \%)$ are on chemoprophylaxis. Congenital hypertrophic retinal pigment epithelium was detected in 15. Desmoids tumours (6\%) and other extraintestinal manifestations including osteomas, sebacious cysts and dental abnormalities (34\%) were also detected. A thyroid gland malignancy was screen detected while retinoblastoma, hepatoblastoma and cerebral tumours were seen in pedigrees.

Conclusions A polyposis register may improve prognosis of FAP by early detection. It will help coordinate, optimise and streamline clinical management of patients with FAP and their relatives at risk.

Ceylon Medical Journal 2011; 56: 66-69

\section{Introduction}

Familial adenomatous polyposis is a rare but an important preventable cause of colorectal malignancy in young adults [1]. It is a dominantly inherited genetic disorder caused by a genetic mutation in the adenomatous polyposis coli (APC) gene in the fifth chromosome, which results in hundreds to thousands of adenomatous polyps throughout the colon and rectum [2]. These polyps usually present in the second decade of life, become symptomatic by the third decade and invariably undergo malignant transformation by the fourth decade [3]. This syndrome is associated with gastro-duodenal polyps and cancer, osteomas, sebaceous cysts, desmoids tumours, congenital hypertrophic retinal pigment epithelium (CHRPE) and extra intestinal malignancies including hepatoblastomas, thyroid tumours, adrenal tumours and brain tumours (Turcot syndrome) $[4,5]$.

Colorectal cancer was the sixth commonest malignancy with an age standardised rate of 3.6 per 100000 population in theyear 2005 in Sri Lanka [7]. Familial adenomatous polyposis accounts for $1 \%$ of this cancer mortality [8]. The incidence of FAP is between 1 in 8300 and 1 in 14025 live births affecting both genders equally, with a uniform worldwide distribution [8]. However, data and information about FAP amongst Sri Lankans is scant.

Once a diagnosis of FAP is established, treatment is definitive and follow up is life-long which involves family members at risk too. The responsibility of examination of the entire family as well as the management of diagnosed patients rests with the physician making the initial diagnosis. The burden may overwhelm the physician and the continuity of care and control of the disease may be lost. Therefore the management should be multidisciplinary and should be undertaken systematically by dedicated and experienced health care personnel to ensure continuity of care.

Lockhart-Mummery published the first surgical account of familial adenomatous polyposis and positive effects of centralised registration from the experience of the first polyposis register at St. Marks Hospital in London in 1925 [9]. A familial adenomatous polyposis registry centralises the information regarding diagnosed FAP patients and at risk family members allowing management of newly diagnosed, treated and family members at risk in

${ }^{1}$ Department of Surgery, University of Kelaniya, and ${ }^{2}$ Ophthalmology Unit, North Colombo Teaching Hospital, Ragama, Sri Lanka.

Correspondence: KUAD, e-mail <udalpatadu@gmail.com>. Received 21 October and accepted 11 December 2010. Competing interests: none declared. 
a systematic manner. A significant improvement in the prognosis of FAP patients has been observed since the establishment of FAP registries worldwide [9-12].

The aim of this paper is to describe an initial experience with monocentric FAP registry and to increase awareness about FAP amongst health care personnel in Sri Lanka. We envisage that this experience will help establish a centralised national FAP register.

\section{Methods}

We established a polyposis register through the following stages: ascertainment of probands, construction of pedigrees, prophylactic screening, treatment and follow up [13].

\section{Ascertainment of probands}

All patients with FAP at North Colombo Teaching Hospital were identified using operative surgery registers, endoscopic registers and clinic notes. A proforma was constructed and patients were invited for interview to gather more information.

\section{Construction of pedigrees}

Genealogical information about index cases and family members was obtained and the pedigrees constructed based on standard criteria for pedigree charts [13].

\section{Identification of family members at risk}

Family members who were at risk were identified using the information from the constructed pedigrees and those who consented were registered using a structured proforma. The relatives were invited for screening.

\section{Screening of members at risk}

Data of all probands and family members at risk were acquired and entered into an electronic database for easy access and review. A structured consent form was created in all three languages, which each registry participant completed to give consent for screening. This ensured individual trust and privacy whilst imparting necessary details regarding themselves and their relatives. Surveillance of the large intestine with flexible sigmoidoscopy was commenced at twelve years of age. Polyps, if found, were excised and sent for histology. In the presence of hundreds of polyps, those that were 10 $\mathrm{mm}$ or more in diameter were removed. Those with polyps were scheduled for total colonoscopy to map the extent of their polyposis and to exclude cancer. Ophthalmological screening for CHRPE was performed by a consultant ophthalmologist with slit lamp examination after dilating both pupils. Blood samples of patients $(3 \mathrm{ml})$ were drawn, centrifuged and stored at $-70^{\circ} \mathrm{C}$ for genetic testing.

Statistical analysis was performed using the $\chi^{2}$ test. A $p<0.05$ was considered significant. Approval was obtained from the Ethical Review Committees of the National Research Council and the University of Kelaniya (NRC 05-56).

\section{Treatment and follow up}

Once diagnosed, a management plan was drawn depending on the mode of presentation, pattern of polyposis, age and patient's consent. This included either chemoprophylaxis or proctocolectomy. For those patients with FAP induced colorectal cancer, staging was undertaken as standard and oncological referral and post surgical follow up with upper gastro intestinal endoscopy (UGIE), pouchoscopy and carcinoembryonic antigen levels were planned [14].

A plan of follow up was also drawn up for family members at risk and those patients already on chemoprophylaxis. For those who had had proctocolectomy with an ileal pouch or colectomy with an ileorectal anastomosis, frequent surveillance of the ileal pouch or the rectum, respectively, was scheduled. An information booklet, available in English, Sinhala and Tamil was distributed amongst all registered individuals to help improve their knowledge about the disease and to disseminate information amongst family members. FAP registry staff made themselves available to registrants for further information and whenever a problem arose through either a texting service on a mobile or by direct telephone contact.

\section{Results}

Since its establishment in December 2009, the polyposis register has enrolled 27 probands (12 male and 15 female, age 11 - 52 years, median age 34 years). Pedigree analysis showed 46 suspected or diagnosed FAP patients and 206 family members who may be at risk of familial polyposis. The number of registered individuals was 51 . Of these, thirty five were diagnosed FAP patients ( 17 male and 18 female). Geographic distribution showed that patients came from all parts of the country.

Most of the pedigrees showed an autosomal dominant pattern while $1(3.7 \%)$ showed an autosomal recessive pattern suggesting attenuated polyposis. Eleven probands did not give a previous family history of FAP or cancer, suggesting a spontaneous mutation rate of $41 \%$. Desmoid tumours were seen in $2(7 \%)$ patients and extra intestinal manifestations like osteomas, sebaceous cysts and dental abnormalities were seen in 12 (34\%). Extra intestinal malignancies observed in pedigrees included retinoblastoma $(n=1)$, hepatoblastoma $(n=1)$ and cerebral tumours $(n=2)$. A papillary carcinoma of the thyroid was diagnosed in one patient on screening.

CHRPE was undertaken in 30 individuals. Of these, 15 were found to have CHRPE lesions. Ten (67\%) individuals had bilateral lesions. CHRPE was present in $13(76 \%)$ of 17 diagnosed FAP patients. 
The distribution of polyps showed left sided predominance $(73 \%)$ with tubular adenomas and tubulovillous adenomas occurring in similar frequency. Twenty seven (77\%) diagnosed patients were symptomatic with most presenting with rectal bleeding or altered bowel habits. Two patients had features of intestinal obstruction and concomitant cancer necessitating urgent surgical intervention. The median age of the symptomatic group was 34 years (11-52 years). Seventeen patients (63\%), amongst the symptomatic group, were diagnosed as having concomitant colorectal cancer. Most were stage 2 cancers while 3 patients had stage 3 cancer based on American Joint Committee on Cancer (AJCC) criteria [15]. By comparison, the screen detected group had only one (13\%) concomitant stage 1 cancer $(p=0.015)$. The median age of the screen detected group of FAP was 32 years (12-48 yrs) (Figure 1).
Twenty seven registered patients (73\%) had undergone surgery. Restorative procto colectomy was performed in twenty five while two have undergone subtotal colectomy and an ileostomy. Eight (23\%) patients are currently on chemoprophylaxis. The duration of chemoprophylaxis with sulindac has ranged from two months to two years.

Upper gastro intestinal surveillance revealed gastroduodenal polyps in 15 patients (42\%) which were categorised according to the Spigelman criteria (16, Table 1). Two patients (9\%) have had gastric carcinoma. Those who were at high risk - Spigelman stage 3 were commenced on chemoprophylaxis while patients with gastric carcinoma underwent total gastrectomy.

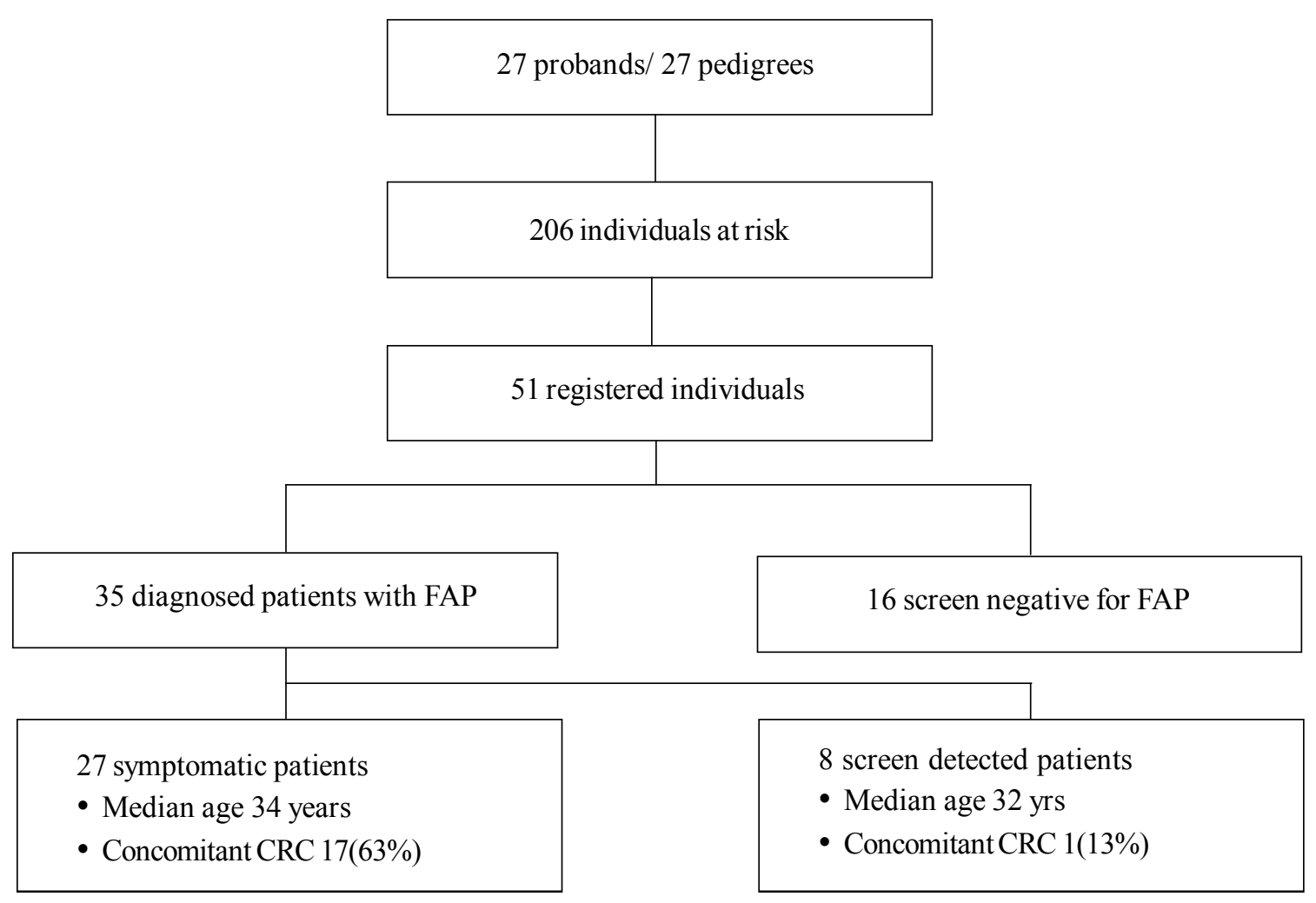

Figure 1. FAP registry at a glance

CRC: colorectal cancer

Table 1. Spigelman classification

\begin{tabular}{cccll}
\hline Points & Polyp number & Polyp size $(\mathrm{mm})$ & Histology & Dyplasia \\
\hline 1 & $1-4$ & $1-4$ & Tubular & Mild \\
2 & $5-20$ & $4-10$ & Tubulovillous & Moderate \\
3 & $>20$ & $>10$ & Villous & Severe \\
\hline
\end{tabular}

Stage I, 1-4 points; Stage II, 5-6 points; Stage III, 7-8 points; Stage IV, 9-12 points 


\section{Discussion}

We believe that this is the first polyposis registry for FAP in Sri Lanka. FAP is a rare but complex clinical entity, which requires multidisciplinary management involving counselling, genetic testing, clinical screening, prophylactic treatment, management of family members and follow up. Therefore a coordinated effort is needed which may be achieved via a polyposis registry [17].

CHRPE is one of the earliest lesions to manifest and is reported to occur in about ninety percent of affected individuals [18]. In our study CHRPE was positive in seventy six percent. As an adjunctive screening tool, it is relatively cheap, non invasive and may bear less of a personal stigma in a socially sensitive culture, such as ours. Our experience has been that identification of CHRPE lesions has encouraged individuals to undergo screening by colonoscopy in diagnosis of FAP, helped intensify screening of relatives at risk and improved compliance of asymptomatic persons who may be at risk. It has also been reported that the presence of CHRPE may be a means of targeted genetic screening [19].

A comprehensive management plan was drawn for each of the participants in the FAP registry including long term follow up. For others, follow up schedules were drawn up until such time that genetic testing is made available to exclude FAP. In addition to educational literature, a familial polyposis registry web site is being planned to optimize the management of these individuals, for ease of access to information.

\section{Conclusion}

The management of familial adenomatous polyposis is essentially multidisciplinary. A polyposis register will improve prognosis by early detection and by allowing prophylactic treatment which would optimise clinical management of FAP patients. A national polyposis register will be of great benefit to improve early diagnosis of individuals at risk and to increase awareness among relatives and healthcare workers in Sri Lanka.

\section{Acknowledgements}

Other members of the polyposis registry are Mr. I. G. R. Kumararathne and Ms. Thakshila Mallikarachchi.

\section{References}

1. Campbell WJ, Spence RA, Parks TG. Familial adenomatous polyposis. British Journal of Surgery 1994; 81: 1722-33.

2. Kinzler KW, Nilbert MC, Su LK, et al. Identification of FAP locus genes from chromosome 5q21. Science 1991; 253: $661-5$.

3. Petersen GM, Slack J, Nakamura Y. Screening guidelines and premorbid diagnosis of familial adenomatous polyposis using linkage. Gastroenterology 1991; 100: 1658-64.
4. Lyons LA, Lewis RA, Strong LC, et al. A genetic study of Gardner syndrome and congenital hypertrophy of the retinal pigment epithelium. American Journal of Human Genetics 1988; 42: 290-6.

5. Hamilton SR, Liu B, Parsons RE, et al. The molecular basis of Turcot's syndrome. New England Journal of Medicine 1995; 332: 839-47.

6. World Health Organization, Cancer, February 2009, http:// www.who.int /mediacentre/factsheets/fs297/en, accessed February 2009.

7. National Cancer Control Programme - 2009. Cancer incidence data: Sri Lanka year 2001-2005, Cancer Registry Sri Lanka 2009.

8. Wennstrom J, Pierce ER, McKusick VA. Hereditary benign and malignant lesions of the large bowel. Cancer 1974; 34: $850-7$.

9. Lockhart-Mummery P. Cancer and heredity. Lancet 1925; 1: 427-9.

10. Bülow S. Results of national registration of familial adenomatous polyposis. Gut 2003; 52: 742-6.

11. Vasen HFA, Riffioen G, Offerhaus GJA, et al. The value of screening and central registration of familial adenomatous polyposis: a study of 82 families in the Netherlands. Diseases of the Colon and Rectum 1990; 33: 227-30.

12. Ho JW, Chu KM, TSE CW, Yuen ST. Phenotype and management of patients with familial adenomatous polyposis in Hong Kong: perspective of the Hereditary Gastrointestinal Cancer Registry. Hong Kong Medical Journal 2002; 8: 342-7.

13. Stern HS. The Canadian Familial Adenomatous Polyposis Registry: past, present and future. Journal of the Royal Society of Medicine 1996; 89: 153-4.

14. Chan KK, Dassanayake B, Deen R, et al. Young patients with colorectal cancer have poor survival in the first twenty months after operation and predictable survival in the medium and long term: analysis of survival and prognostic markers. World Journal of Surgical Oncology 2010; 8: 82.

15. Edge SE, Byrd DR, Carducci MA, Compton CC, eds. AJCC Cancer Staging Manual. 7th ed. New York: Springer, 2010.

16. Spigelman AD, Williams CB, Talbot IC, Domizio P, Phillips RK. Upper gastrointestinal cancer in patients with familial adenomatous polyposis. Lancet 1989; 2: 783-5.

17. Bulow S, Burn J, Neale K, Northover J, Vasen H. The establishment of a polyposis register. International Journal of Colorectal Disease 1993; 8: 34-8.

18. Chen CS, Phillips KD, Grist S, et al. Congenital hypertrophy of the retinal pigment epithelium (CHRPE) in familial colorectal cancer. Familial Cancer 2006; 5: 397-404.

19. Lyons LA, Lewis RA, Strong LC, et al. A genetic study of Gardner's syndrome and congenital hypertrophy of the retinal pigment epithelium. American Journal of Human Genetics 1988; 42: 290-6. 